\title{
Estructura y agencia en la migración infantil centroamericana ${ }^{1}$
}

\section{Guillermo E. Acuña González²}

\section{Resumen}

Recepción: 24 de junio de 2015 / Aprobación: 6 de octubre de 2015

Al promediar el año 2014 se activaron voces que alertaban sobre la presencia de decenas de miles de niños y niñas migrantes centroamericanos tratando de cruzar la frontera entre México y Estados Unidos. Las respuestas elaboradas por las autoridades migratorias y estatales de ambos países privilegiaron un esquema de seguridad y control, sin atender las especificidades de esta migración. Los discursos públicos homogeneizaron las miradas sobre quienes hacían parte de estos procesos migratorios e invisibilizaron sus rasgos específicos. Desde un análisis que recupera las dimensiones de estructura y agencia, el presente artículo aborda las causas y motivaciones en la decisión de migrar de los niños y niñas migrantes centroamericanos que se dirigen a Estados Unidos y reflexiona acerca de la importancia de tomar en cuenta las especificidades que presentan dichos actores en la migración.

\section{Palabras clave}

Migración; niñez; violencia; agencia; estructura

\section{Abstract}

In mid-2014, there were suddenly voices alerting of the presence of tens of thousands of Central American migrant children trying to cross the border between Mexico and the United States. The responses of immigration and state authorities in both countries privileged topics of security and control, rather than addressing the specifics of this migration. Public speeches homogenized the views on those who were part of these migration processes and erased their specific features. From the dimensions of structure and agency, this article discusses the causes and motivations in the Central America children's decision to migrate to the United States, and examines the importance of taking into account the specificities of these actors in migration.

\section{Key Words}

Migration; childhood; violence; agency; structure

1 El autor de este artículo agradece el apoyo de Mónica Fallas, internacionalista costarricense y actual funcionaria de la organización regional Alianza para las Migraciones en Centroamérica y el Caribe (CAMMINA), quien proporcionó información documental valiosa para su elaboración.

2 Costarricense. M.Sc. en Comunicación por la Universidad de Costa Rica. Director del Instituto de Estudios Sociales en Población (IDESPO) de la Universidad Nacional (UNA). Correo electrónico: guillermo.acuna.gonzalez@una.cr 


\section{Resumo}

Durante o ano passado, 2014, elevaram-se vozes de advertência sobre a presença de centenas de milhares de crianças da América Central tentando cruzar a fronteira entre o México e os Estados Unidos. Nas respostas apresentadas por imigração e pelas autoridades federais de ambos países, as aborda gens foram basea da semsegurança e controle, sem considerar as necessidades específicas dessa população migrante especial. Os discursos públicos concentraram sua atenção sobre aqueles que fizeram parte deste processo de migração, tornando invisíveis as características específicas dessa população. Este artigo aborda as causas e motivaçõ es na decisão de meninos e meninas da América Central de migrar para os Estados Unidos. A partir de uma análise que recupera as dimensões de estrutura e agência, o artigo reflete sobre a importância de ter emconta as características específicas que apresenta messes atores específicos neste processo.

\section{Palavras chave}

Migração; infância; violência; agência; estrutura

\section{Introducción}

n el documental Wich Way Home (Rebeca Camissa, 2009), le preguntan a Kevin, un niño hondureño de 13 años, por los riesgos a los que se enfrentaría en caso de seguir su viaje hacia Estados Unidos montado sobre "la bestia", el tren de carga que atraviesa territorio mexicano y que cotidianamente lleva sobre sus lomos cientos de personas migrantes, la mayoría de ellos de origen centroamericano ${ }^{3}$. La travesía presenta riesgos, peligros y oportunidades. Kevin construye un sueño y para lograrlo se hace acompañar de un amigo, también hondureño y de otros niños mexicanos que se unen en el trayecto, para formar una pequeña red migratoria con un solo propósito: llegar hasta la frontera norte e intentar cruzarla. A mitad del camino y ante una pregunta que le realiza la producción del documental sobre cómo se imagina ese paso de la frontera entre México y Estados Unidos, Kevin responde con una contundente afirmación: "Está cabrón, pero vamos a pasar", refiriéndose a las dificultades propias de la geografía fronteriza y la presencia en esta de actores que vulneran constantemente la situación de las personas migrantes. De acuerdo con Ramírez Parra, el principal factor de riesgo para los niños y niñas migrantes es su edad, pues tienen acceso a

3 Según el documental, el niño parte de su casa principalmente motivado por ayudar a su mamá a salir de la condición de pobreza en la que se encuentran. En apariencia, no medió consulta ni autorización expresa de su madre para que intentara el viaje, por lo que es posible observar una persona con una determinación concreta que lo lleva a tomar la decisión. Justamente la preocupación de su madre sobre las pocas oportunidades de estudio y trabajo que le ofrece el entorno inmediato, la lleva a asegurar que Kevin pruebe suerte en Estados Unidos, es mejor a que se quede con las posibilidades limitadas en su país. La desconexión del niño de su entorno familiar y comunitario es quizá uno de los impactos más fuertes observados en esta historia. 
menos recursos y enfrentan de forma desigual las redes de poder y dominio (2003, p. 4).

La salida de población desde Centroamérica hacia Estados Unidos revela causas de naturaleza estructural (desigualdad, pobreza, desprotección y exclusión institucional) vinculadas a motivaciones individuales, que, al igual que el niño del documental, impulsan a miles de niños y niñas centroamericanos a salir de sus contextos de origen. Más allá de una actuación impensada e impulsiva, los contextos económico, social, cultural, familiar e institucional actúan como determinantes para tomar la decisión de emprender el viaje migratorio. Activan una serie de motivos, recursos y herramientas personales que se ponen en funcionamiento como estrategia para el desarrollo de la travesía migratoria. En los meses de junio, julio y agosto del año 2014, medios de comunicación regionales y globales aumentaron la cobertura de noticias sobre la situación experimentada por miles de niños y niñas migrantes no acompañados, la mayoría de ellos provenientes de los países del norte de la región centroamericana (Guatemala, El Salvador y Honduras), identificados en distintos puntos de la frontera entre México y Estados Unidos ${ }^{4}$. Esta situación "llevó al establecimiento de refugios improvisados en poblados, incluso en bases militares, en Texas y California, y la detención de millares de niños a veces en condiciones de hacinamiento" (Carreño Figueras, 8 de junio de 2014).

En los últimos años, la migración de niños y niñas, junto con el aumento de la migración femenina, representa una de las tendencias novedosas en el marco de la movilidad humana observada en la región. Pese a la incidencia, el interés por la migración de niños y niñas sigue siendo escaso y, a menudo resultan invisibilizados en los análisis sobre la migración regional y negados como personas con derechos, como bien señala Vennet:

Son invisibles y excluidos porque están ausentes de los estudios de migración; son muy recientes los estudios que abordan los niños, niñas y adolescentes migrantes; están perfectamente ausentes de las políticas; no aparece nunca la palabra niñez en las políticas migratorias. Generalmente están también ausentes de las políticas de desarrollo; hay una enorme falta de programas específicos para la atención de sus necesidades y derechos. No son escuchados ni tomados en cuenta, eso es un problema grave; hay una visión muy conservadora y equivocada de la niñez; se cree que no son capaces de pensar en cuál puede ser la mejor opción para su vida, ni opinar sobre su destino, pero sí lo son (Vennet, 2006, p. 5).

El presente artículo aborda el desarrollo reciente de la niñez migrante en la región centroamericana, desde una reflexión sobre la relación entre agencia y los procesos estructurales que producen su expulsión. Se toma como punto de partida el marco coyuntural denominado "crisis de niños y niñas migrantes

4 Por ejemplo, la cadena global CNN, en su edición en español, presentó en junio de 2014 un reportaje titulado "La realidad de los niños y niñas migrantes que buscan cruzar la frontera de Estados Unidos", en el que describía la situación de emergencia por el grado de vulnerabilidad al que se encontraban expuestos. 
no acompañados", declarado así durante la mitad del año 2014. Incluye una reflexión sobre los desafíos que el estudio de las migraciones de niños y niñas imponen tanto para la academia como para las instituciones y organizaciones involucradas en el abordaje de la problemática.

\section{Estructura y agencia en la migración de niños y niñas centroamericanos}

Las migraciones internacionales forman parte de fenómenos globales con importantes efectos en las personas y los territorios implicados. Como lo ha apuntado ya Saskia Sassen (2010), "la nueva fase del capitalismo avanzado se caracteriza por un marcado aumento del número de personas que han sido expulsadas" (Sassen, 15 de junio 2010). Se reconoce el fenómeno migratorio desde una mirada global, en el que procesos y actores forman parte del escenario. En este contexto, al analizar la estructura y agencia en los procesos migratorios se abordan las relaciones y tensiones que se producen entre la persona migrante y los procesos sociales, económicos, culturales e institucionales ante los cuales interactúa, tanto en los contextos de origen como de destino. En el caso centroamericano, los determinantes presentes en las sociedades de origen son factores que explican la salida de un buen porcentaje de población hacia otros países de la región o Estados Unidos, particularmente. En la presente reflexión se toma como punto de partida las nociones elaboradas en algunos estudios migratorios, tales como los de Brettel (2002) y Bakewell y otros (2009).

De acuerdo con Brettell, la estructura hace referencia a limitaciones o barreras existentes en las sociedades de origen y destino, que condicionan la vida de las personas migrantes, en tanto que la agencia del individuo lo impulsa a tomar decisiones frente a tales limitaciones, en ambos contextos (origen y destino) (2002, p. 431). Las personas migrantes se ubican ante los procesos estructurales (de naturaleza económica, política, cultural, etcétera) y las políticas nacionales, determinando e influyendo en los flujos migratorios. Bakewell, Castles, Jonsson y Vezzoli (2009), por su parte, plantean que la migración no puede ser separada de procesos de cambio social y económico que alteran la distribución espacial de la estructura de oportunidades y los patrones de movilidad. Indican que es erróneo reducir a las personas migrantes como actores pasivos frente a las fuerzas macro que interactúan a escala global.

Desde estas interpretaciones, una posible explicación al aumento de la migración de niños y niñas centroamericanos tiene que ver con los rasgos expulsores de sus contextos locales/nacionales de origen y los factores de atracción en la sociedad de destino, aunado a los marcos de transformación de las políticas migratorias en Estados Unidos, que propiciaban, al menos en sus inicios, la posibilidad de una más expedita forma de regularización de los cerca de 11

46 millones de personas migrantes que permanecían en aquel país en condiciones 
migratorias irregulares. Esta medida impactaría a sus familiares, situación que provocó un considerable aumento de flujos de niños y niñas que buscaban reencontrarse con sus padres y permanecer en aquel país.

El enfoque aquí planteado busca otorgar importancia al actor como protagonista de sus acciones. De acuerdo con Anna Sanmartín, si bien hay factores estructurales en los escenarios de emisión y recepción, es importante anotar la actuación de la persona migrante en tales escenarios.

Sería un error reducir la migración a la figura de un títere pasivo que se mueve ante macro-fuerzas que la distribuyen por el globo. La gente tiene agencia, actúa, decide y precisamente por ello, su movilidad es también una fuerza en potencia para el cambio estructural (Sanmartín, 2010, p. 3).

Por su parte, Saraví señala que ante la pérdida en los referentes de sentido como la escuela y el trabajo, instituciones en crisis, los niños, niñas y jóvenes de la región diseñan estrategias entre las que encuentran las migraciones y la evasión a esos marcos estructurales (2009, p. 59). Actúan como personas con disposición para plantearse respuestas ante dichos contextos ${ }^{5}$. Desde este enfoque, la connotación a ellos como "niños y niñas migrantes no acompañados" (unaccompanied children, en su definición en inglés) resulta discutible, más allá de las motivaciones que les provocan hacer la travesía viajar sin la compañía de sus familiares o parientes más cercanos. Esta aseveración, supone una persona aislada de su entorno inmediato, actuando de forma individual.

En este artículo se parte del reconocimiento formal de la migración de niños y niñas no acompañados (es decir, quienes están separados de ambos padres y otros parientes y no están al cuidado de un adulto al que, por ley o costumbre, incumbe esa responsabilidad) ${ }^{6}$ y se incluye también una serie de consideraciones acerca de la acción de actores irregulares, el establecimiento de vinculaciones temporales de solidaridad y acompañamiento junto con otros niños, niñas y personas adultas migrantes, a quienes se unen para realizar la travesía migratoria por territorio mexicano, principalmente. Desde este planteamiento sobre las relaciones entre los procesos estructurales e individuales en la migración de niños y niñas centroamericanos es posible advertir las paradojas que se producen y que los conectan-desconectan con suma facilidad de los marcos de inclusión social, institucional, cultural y simbólica en los países de origen, tránsito y destino. Como lo señala un informe sobre la juventud en Iberoamérica, en medio de cambios estructurales que les proveen de mayor

$5 \quad$ Sobre la capacidad de los niños, niñas y jóvenes migrantes, el sacerdote Alejandro Solalinde, Director del albergue Hermanos en el Camino de Ixtepec, Oaxaca, y Coordinador de la Pastoral de Movilidad Humana Pacífico Sur del Episcopado Mexicano señala: "Los jóvenes para mí son la fuerza, pero no sólo la fuerza, son también preparación, son sensatez, ideales, solidaridad; una promesa, que ya no es futura, son una realidad. Si se organizan ellos, lo van a poder hacer" (Márquez, 2014, p. 193).

6 Comité de los Derechos del Niño: Observación general № 6 (2005) Trato de los menores no acompañados y separados de su familia fuera de su país de origen, $39^{\circ}$ período de sesiones, $\mathrm{CRC/}$ GC/2005/6, $1^{\circ}$ de septiembre de 2005, párrafo 7, citado en ACNUR-OIT-OIM-UNCEF, 2013. 
autodeterminación y protagonismo, pero también de precariedad y desmovilización (CEPAL, 2007, p. 20).

Finalmente, el análisis debe incluir una diferenciación entre los factores causales y los motivos de la migración. El primer caso incluye dinámicas como la pobreza, la falta de infraestructura productiva, el deterioro de los recursos naturales, la falta de oportunidades de empleo, la carencia de servicios públicos y el crecimiento demográfico. Entre tanto, las motivaciones aluden a los factores estrictamente individuales y subjetivos, entre los que se encuentran la búsqueda de un familiar o hermano, el deseo de conocer nuevos lugares, el deseo de vivir nuevas experiencias, el maltrato, la violencia y la soledad (Ramírez Parra, 2003, pp. 4-5).

\section{Migración de niños y niñas centroamericanos hacia Estados Unidos: el año 2014 como coyuntura}

Los niños y niñas migrantes centroamericanos son actores de procesos migratorios mucho más amplios que se dirigen a países del interior de la región (migración intrarregional) y hacia el norte de la misma (migración extraregional), en particular Estados Unidos. Muchos de estos flujos se desarrollan en condiciones de indocumentación e irregularidad migratoria, por lo que aumenta la exposición y el riesgo, producidos en muchos casos por actores del crimen transnacional organizado y las mismas autoridades ${ }^{8}$. Serán además víctimas de la insuficiencia de las políticas de protección de gobiernos de origen, tránsito y destino de la migración.

En el proceso experimentan desintegración de sus contextos de origen, los riesgos y amenazas vividos en el tránsito (violencia, asaltos, trata y tráfico de personas) y el desarraigo en el lugar de destino. En este último espacio viven diversas formas de negación de sus derechos -por ejemplo, a la salud, la educación, el esparcimiento, la identidad propia, la identidad cultural, la explotación(Organización Internacional para las Migraciones; Fondo de Naciones Unidas para la Infancia \& Organización Internacional del Trabajo, 2013, p. 20). Sus rutas son amplias y diversas. La diversidad de trayectos por medio de los cuales intentan llegar a su objetivo, muestra realidades complejas frente a las cuales establecen respuestas y estrategias.

7 La migración intrarregional se produce entre países de la región centroamericana, con un alto componente laboral, aunque también se identifican otros determinantes como la búsqueda de servicios de salud adecuados, por ejemplo. Los principales flujos al interior de los cuales se incluyen niños y niñas migrantes son los que se producen entre Panamá y Costa Rica, entre Nicaragua y Costa Rica, entre Nicaragua y El Salvador, y entre El Salvador, Honduras y Guatemala (OIM, 2013, p. 28).

8 La mayoría de violaciones a los derechos humanos de esta población migrante son cometidas por las personas que tendrían que garantizar su seguridad a lo largo del proceso, tales como las autoridades migratorias y los agentes de policía (Escobar Sartí, 2008, p. 84). 
Por país, se puede observar que los provenientes de Honduras establecen dos vías de movilidad. La primera, utilizada con mayor frecuencia está marcada por el cruce hacia Guatemala por el cruce de El Corinto, y la segunda por Agua Caliente. Entre las dos, Corinto registra más del 90\% de los cruces de los NNAS (niños, niñas y adolescentes) provenientes de aquel país. Por su lado, El Salvador registra el mayor número de traslados por el cruce La Hachadura. Los itinerarios de migración señalan el derrotero que determinará el punto de cruce que realizarán en la frontera entre Guatemala y México. En todos los casos los desplazamientos son terrestres, utilizando mayormente autobuses (88\%), carros particulares (5\%) o pequeñas furgonetas de pasajeros llamadas "combis" (5\%). Únicamente se registraron dos casos de NNAS varones quienes viajaron solicitando transporte gratuito a los conductores de tráileres de carga, y otros casos donde los NNAS fueron alternando diversos medios de transporte para llegar a la frontera con México. De manera, general el viaje desde su lugar de origen hasta la frontera con México se realiza normalmente en un día (75\%), e incluso en unas cuantas horas (22\%) (Camargo, 2014, p. 51).

Muestran una gran diversidad en sus características y sus perfiles. Se trata de niños y niñas ${ }^{10}$ campesinas, indígenas, representantes de la diversidad sexual, provenientes de comunidades afrodescendientes, de la ciudad y del campo centroamericano, entre otros. Su heterogeneidad es justamente uno de los rasgos de la agencia del individuo presente en la coyuntura de análisis, pese a que fue insistentemente anulada por narrativas dominantes que sopesaron el volumen y la cantidad.

Desde sus características diversas y específicas enfrentan visiones, prácticas y actitudes que los agregan a esas lecturas predominantes sobre el migrante como el otro, el distinto, el raro, el extranjero. En definitiva, el extraño. Su tránsito por zonas de frontera, comunidades, localidades, albergues, sitios públicos no pasa desapercibido, ni para las poblaciones de tránsito y destino, ni para los medios de comunicación, ni para las autoridades. Ponen en perspectiva percepciones sobre un mundo distinto, se enfrentan a estereotipos respecto a su color de piel, su forma de hablar, vestir y relacionarse. No en pocas oportunidades se expondrán a valoraciones que los criminalizan, como lo señala Vennet:

También hay una tendencia, cuando hablamos de su participación en los flujos migratorios, a que se dé una criminalización en la opinión pública; los adolescentes migrantes son "Maras", son delincuentes, son chicos de la calle, se prostituyen, roban, asaltan y hay una tendencia a penalizarlos por emigrar, poniéndolos en detención, detrás de las rejas, o también hay una tendencia actualmente a querer proponer en el caso de los que reinciden en su movimiento migratorio que sean castigados o que sus familias sean penalizadas, lo que obviamente no va a ser

$9 \quad$ Los porcentajes fueron obtenidos en la consulta con niños y niñas migrantes centroamericanas realizada en el marco del estudio de Camargo.

10 Existe muy poca precisión en la literatura consultada acerca de las edades detectadas durante el periodo en estudio. Ramirez Parra (2010) aventura un perfil para el caso de los niños y niñas en zonas de frontera entre México y Guatemala, en el que el rango etareo se ubica entre los 11 y los 17 años. 
ninguna solución, y hay una falta de acceso efectivo a la protección básica e integral de sus derechos, sean básicos, pero también económicos, sociales y culturales (Vennet, 2006, p. 5).

Durante los meses de mayo, junio y julio de 2014 fue notable la presencia de un significativo número de niños y niñas migrantes centroamericanos en diferentes zonas de la frontera entre México y Estados Unidos, particularmente en estaciones migratorias, albergues para migrantes asentados, centros de detención y espacios públicos como centrales de buses, mercados o plazas públicas. Sin embargo, no se trató de un hecho nuevo, pues en años anteriores ya se consideraba la presencia de niños y niñas en los flujos migratorios que se movilizaban hacia Estados Unidos, principalmente. Su aumento en los flujos migratorios regionales se empezó a observar durante los años de la década del 2000, particularmente en el territorio mexicano como zona de tránsito para esta migración (Ramírez Parra, 2003; Sin Fronteras, 2005). Por ejemplo, Ramírez Parra menciona cómo se empezaron a observar niños y niñas en los grupos de migrantes centroamericanos en la frontera sur de México, particularmente en el Soconusco, Chiapas. De acuerdo con la autora, viajaban solos, encargados por sus familiares a las redes de polleros o coyotes, o se perdían en el camino (Ramírez Parra, 2003, p. 2). En este sentido, se trata de una tendencia con algunos años de desarrollo, en los que los flujos migratorios regionales empezaron a mostrar evidencias de estos nuevos actores, motivados en primera instancia por el deseo de reunificación familiar en Estados Unidos.

La primera década del 2000, también habían sido identificados factores económicos en la movilidad de estas personas hacia fuera de la región. En un estudio sobre migración y trabajo infantil en la región se enfatizaba en el carácter específico de los movimientos migratorios de estas personas, determinado por su articulación temporal o permanente a los mercados de trabajo regionales (Acuña, 2010). Lo novedoso de la reciente coyuntura fue la intensidad de dicha migración. Las autoridades migratorias de Estados Unidos calculaban que hacia finales de 2014 cruzaron la frontera cercan de 60000 niños y niñas de México y los países del denominado triángulo norte de la región centroamericana (Guatemala, Honduras y El Salvador. La prevalencia de estos tres países como origen de las migraciones de niños y niñas centroamericanos es contundente ${ }^{11}$. (Al respecto, puede revisarse OIM, 2013; Camargo, 2014; Orozco y Yansuela, 2014; Ceriani, 2015) $)^{12}$.

11 Esta prevalencia también se expresa en sendas producciones audiovisuales, que al igual que Way Wich Home, retratan el tránsito migratorio desde Centroamérica a Estados Unidos, pasando por México. En el año 2009, la película Sin Nombre, dirigida por Cary Fukunaga, relata la travesía de Sayra, joven hondureña que decide viajar junto con su padre y su tío desde el norte de Honduras para llegar hasta New Jersey, Estados Unidos. Entre tanto, en el año 2013, la película La Jaula de Oro, dirigida por Diego Quemada Diez, muestra el viaje migratorio de unos jóvenes migrantes guatemaltecos junto con un joven indígena tzotzil, en el que experimentan violencias cotidianas en su periplo hacia Estados Unidos. migratorias en Ciudad de México y Tapachula (2014). En este presenta un interesante perfil que bien 
El origen de las migraciones desde estos tres territorios tiene explicaciones diversas, entre las que cabe indicar un déficit importante en las políticas de protección de la niñez por parte de los estados implicados. De acuerdo con el estudio citado de Ceriani (2015) las limitaciones se expresan entre otros aspectos en lagunas normativas, debilidades institucionales, falta de recursos, deficiencia en la implementación de políticas públicas, discriminación y altos niveles de impunidad. El mismo estudio indica una serie de problemáticas que afectan el cumplimiento de los derechos de los niños y niñas:

Pobreza, analfabetismo, deserción escolar, falta de oportunidades, desempleo de adolescentes y jóvenes, formas variadas y crecientes de violencia (social, institucional, crimen organizado, violencia de género y violencia doméstica); impunidad frente a estos hechos de violencia, trabas en el acceso a la justicia, altos índices de corrupción, connivencia entre organismos públicos y actores vinculados al crimen organizado, políticas de institucionalización de niños y niñas en situación de vulnerabilidad, sistemas de bienestar infantil disfuncionales y asistencialistas, separación de los padres, déficit habitacionales y sanitarios, desigualdad de género y con base en el origen étnico (población indígena), explotación y trabajo infantil, trata de personas, entre otros indicadores, revelan un nivel considerablemente preocupante de privación de derechos básicos de la niñez (Ceriani, 2015, pp. 37-38).

Solo de octubre de 2013 a julio de 2014 el número de niños y niñas no acompañados provenientes de El Salvador, Honduras y Guatemala había alcanzado la cifra de 48000 (Camargo, 2014, p. 20). El constante flujo en zonas de frontera, condujo a actores vinculados con la migración, entre los que destacan autoridades migratorias de Estados Unidos y México, medios de comunicación y organizaciones sociales de asistencia a personas migrantes, a denominar la situación como "crisis" y declarar una estrategia de emergencia humanitaria para abordar de manera inmediata la problemática ${ }^{13}$. Además, se realizó una solicitud de fondos de emergencia por 3700 millones de dólares por parte de la administración Obama para crear nuevos centros de detención, más jueces migratorios y fondos de repatriación de migrantes, todo con el propósito de contener la "crisis" (Castañeda, 15 de julio de 2014); tal solicitud

podría ser utilizado como referencia para caracterizar esta población: se presenta un ligero predominio de niños por sobre las niñas para los tres países y resalta el hecho de que una mayoría importante saben leer y escribir. Se identificaron rasgos de actividad laboral (labores agrícolas y comerciales) en sus países de origen antes de emprender el viaje y solo un tercio se dedicaba a estudiar. Un 15\% aseguró hablar alguna lengua indígena (mam y quiché guatemalteco) y un 5\% de ellos indicó ser padre de familia, predominando los niños y niñas provenientes de Honduras con esta condición (Camargo, 2014, pp. 38-39).

13 Por ejemplo, la organización de defensa de las personas migrantes en Estados Unidos, NALACC, precisaba en un comunicado publicado el 1 de julio de 2014, lo siguiente: "Las imágenes de niños pequeños colgando desesperadamente de trenes en movimiento en México o tendidos en el suelo de centros de detención para jóvenes en Texas, los cuales están sobrepoblados, han sorprendido al mundo. Incluso los cínicos más duros se conmueven ante los rostros de tantos niños en condiciones tan desesperantes. A la espera de que más de 60000 niños intenten cruzar la frontera en el curso del verano, nos encontramos claramente ante una crisis humanitaria urgente. Los recursos públicos y privados parecen completamente abrumados e incapaces de responder adecuada y humanamente a las necesidades de estos niños" (Shannon, 2014, p. 1). 
indica la magnitud adquirida, las percepciones institucionales sobre el tema y las respuestas para abordarlo.

Por su parte, desde organismos de sociedad civil se indicaba que las medidas solo se orientaban a fortalecer acciones de militarización fronteriza, que producirían el aumento de la migración de los niños y niñas por la zona de influencia entre México y Estados Unidos. La utilización indiscriminada de la figura de la deportación para devolver a los niños y niñas a sus países de origen, los tiempos mínimos en la resolución de los casos y la prevalencia de políticas restrictivas y con enfoque de seguridad de frontera, fueron algunas de las acciones utilizadas durante la gestión de la denominada crisis.

Un consorcio de organizaciones de la sociedad civil de México, Centroamérica y Estados Unidos que trabajan en niñez y adolescencia en migración, se sumó a la preocupación por las estrategias coyunturales. Instaban a los gobiernos de México y Estados Unidos a revisar las políticas migratorias, encaminadas al endurecimiento de la seguridad en las fronteras, generando mayor control y persecución a las personas migrantes. En este marco, hacía una solicitud expresa orientada a garantizar la seguridad de los niños y niñas:

Es imprescindible que los niños y niñas no sean retornados ni deportados automáticamente, ni colocados en centros de detención migratorios. Al contrario, se les debe alojar en albergues que los protejan integralmente y que cuenten con las condiciones apropiadas a sus derechos, y especialmente que posibiliten el derecho a la unidad familiar (CAMMINA, 2014, p. 2).

En los meses de mayor intensidad de la coyuntura afloraron informaciones relacionadas con las deficitarias condiciones que presentaban los sitios donde eran remitidos los niños y niñas identificados y detenidos. Pasaba del lado mexicano, pero también en los espacios de detención dispuestos en Estados Unidos. En este último caso, las quejas contra las patrullas fronterizas y funcionarios de aduanas estadounidenses empezaron a ser frecuentes. Un documento suscrito por organizaciones prodefensa de las personas migrantes identificaba algunas condiciones cercanas a violaciones a los derechos de los niños y niñas migrantes detenidos por autoridades fronterizas en Estados Unidos:

La queja describe a los agentes de la Patrulla Fronteriza negando el cuidado médico necesario para niños tan pequeños como de cinco meses de edad, negándose a proveer pañales para infantes, confiscando y no devolviendo documentos legales y pertenencias personales, profiriendo insultos de índole racista y amenazas de muerte, registro sin ropa y poniéndoles grilletes de tres puntos durante su transportación. Los reportes de tales abusos han sido documentados y reportados durante años, pero no se han implementado reformas, ni se han tomado acciones para hacer que los agentes rindan cuentas (Centro Nacional de Justicia para Inmigrantes, 2014).

Si bien el escenario de coyuntura de la migración de niños y niñas centroamericanos a Estados Unidos está colocado en la frontera entre Estados Unidos y México, no debe omitirse el rol de la frontera sur de México como 
puerta de entrada a flujos migratorios que utilizan este país como zona de tránsito y destino (Ramírez Parra, 2003; Cruz, 2005; Sin Fronteras, 2005; Ceriani, $2012)^{14}$. En estos territorios de frontera, se ha observado durante los últimos años presencia significativa de niños y niñas migrantes centroamericanos, viajando primero como parte de grupos familiares o de personas provenientes de sus mismas comunidades y posteriormente sin la compañía de estos, pero vinculados a redes migratorias de características complejas ${ }^{15}$. También se ha notado el aumento de su participación en el mercado de trabajo fronterizo, como parte de la fuerza de trabajo en fincas cafetaleras ubicadas en Chiapas, como empleadas domésticas, vendedores ambulantes o como parte de actividades de trabajo sexual forzado, producido por las redes ilícitas existentes ${ }^{16}$.

La frontera sur presenta más de 200 rutas por donde transita de forma irregular la migración no documentada que proviene de Centroamérica (Sin Fronteras, 2005, p. 9). Constituye así la antesala a la entrada a Estados Unidos, por lo que su importancia en materia de seguridad y de gestión migratoria resulta fundamental. No en vano, los procesos de control migratorio (rechazo, detención-aseguramiento y deportación) tienen lugar de forma cotidiana y sistemática en diversos puntos de este territorio fronterizo. Las características políticas y jurídicas en la frontera sur, salpican el paso de los niños y niñas migrantes que se dirigen hacia el norte de la región; quedan subsumidos al interior de esquemas de política migratoria basadas en el enfoque de seguridad reactiva, que incorpora el tema del control migratorio desde una perspectiva represiva y contribuye a construir en los imaginarios sociales a los migrantes como enemigos y delincuentes (Sin Fronteras, 2005, p. 10)17.

Ciertamente no es posible separar la coyuntura experimentada en el año 2014, con los marcos de seguridad y los procesos sociopolíticos vividos por

14 En estos territorios, se han identificado diversos tipos de niños y niñas migrantes tales como los migrantes transfronterizos locales, los migrantes fronterizos, los migrantes temporales y los transmigrantes. Este último tipo es el que conforman los flujos identificados recientemente. Viajan solos o en pequeños grupos, contratan coyotes e integran redes sociales que les permiten contender con el viaje migratorio, su estadía en la frontera con Guatemala y el tránsito por México hacia Estados Unidos.

15 Entre los flujos migratorios detectados en la frontera sur mexicana es posible observar residentes fronterizos locales, trabajadores temporales agrícolas y de otros sectores, trabajadoras domésticas, trabajadores en sectores de servicios, transmigrantes entre los que se encuentran hombres, mujeres, niños, niñas, adolescentes y familias en camino hacia Estados Unidos; hombres, mujeres, niñas y niños adolescentes víctimas de trata con fines de explotación sexual comercial, emigrantes chiapanecos que se dirigen al noroeste de México y a Estados Unidos; solicitantes de asilo y refugiados (Sin Fronteras, 2005, p. 11).

16 Carolina Rivera Farfán realizó un acercamiento interesante al trabajo de niños y niñas guatemaltecos en el Soconusco, Chiapas (2011). Entre sus principales reflexiones aborda las deficitarias condiciones laborales en las que son contratados, así como las dinámicas de cruce de fronteras que realizan a diario entre Guatemala y México.

17 Una reflexión aguda sobre el tema de la seguridad, la violencia y los derechos humanos de las personas migrantes en la frontera sur mexicana, puede consultarse en los trabajos de María del Carmen García "Violencia y ética. A propósito de la inmigración irregular y los derechos humanos en la frontera sur de México" (2011) y Daniel Villafuerte Solís "Políticas de seguridad y migración transnacional en la frontera sur de México" (2011). 
la región como parte de las estrategias de control institucional, geográfico y corporal realizados por Estados Unidos, en la denominada "lucha contra las drogas y el narcotráfico". Una lectura crítica realizada por Ted Galen Carpenter, especialista en asuntos internacionales, indicaba que en la discusión sobre la coyuntura de los niños y niñas migrantes no acompañados faltaba el tema de la responsabilidad de Estados Unidos al sostener una lucha prolongada contra las drogas. Por décadas, argumentaba en un artículo de junio de 2014, la cruzada de Washington contra las drogas ilegales destruyó vidas, desestabilizó la sociedad civil e impactó México y la región centroamericana.

\section{Sobre las causas}

Los niños y niñas migrantes experimentan los condicionantes de una región sumida en altos índices de desigualdad social y pobreza, ambientes familiares y comunitarios fragmentados, que generan condiciones adversas para la permanencia en sus países. La situación prevaleciente en los tres principales países desde donde se originan tales procesos migratorios, da cuenta de un conjunto de dimensiones de naturaleza estructural que se imponen en las causalidades prevalecientes. Ceriani (2015) recoge algunos aspectos que a continuación se resumen:

- El Salvador, Guatemala y Honduras ocupan los lugares 115, 125 y 129 en el Índice Global de Desarrollo Humano.

- En Guatemala, de acuerdo con la Encuesta Nacional de Condiciones de Vida (ENCOVI, 2011), el 53.7\% de la población vive en condiciones de pobreza; para el año 2012 el 19\% de los niños y niñas entre los siete y 14 años de edad se desempeñaban en el mercado rural; un $49.8 \%$ de niños menores de cinco años sufren desnutrición crónica (el promedio aumenta si es población infantil indígena a un 56.9\%) y el promedio de escolaridad de los niños y niñas es de solamente de cuatro años.

- El caso de El Salvador también presenta otros datos igualmente alarmantes: en el año 2011 el acceso institucional a centros de educación inicial entre menores de tres años era inferior al 2\%; el porcentaje de niñas, niños y adolescentes con desnutrición global es de 5.5\% y crónica de 19\%.

- Honduras presentaba al año 2011 una incidencia de pobreza en los hogares de un 60\%; el fenómeno se acentúa en el área rural, donde se presentan limitaciones importantes en cuanto a cobertura y calidad de servicios sociales. El promedio de años de estudio escolar de la población de Honduras llega a un $4.3 \%$ regiones rurales y siete años en regiones urbanas.

A propósito de tales datos, no debe obviarse el componente económico, concretamente laboral, que moviliza las migraciones en la región centroamericana. Estos aspectos habían sido analizados en trabajos previos elaborados 54 por quien escribe este artículo (2006, y el citado escrito en el año 2010), en 
los que se daba cuenta de la dinámica laboral vinculada a la movilidad intrarregional de los niños y niñas centroamericanos, con especial atención en los procesos migratorios entre Nicaragua y Costa Rica.

Si bien se indica la reunificación familiar como factor causal de la migración en análisis, es cierto que los procesos de fragmentación familiar también son expresivos de las características de tales entornos. A propósito, es frecuente encontrar niños y niñas migrantes cuya experiencia familiar fue siempre incompleta y, por ejemplo, nunca vivieron con uno de sus padres ya sea porque se había producido una separación temprana o porque el padre o la madre vivían en Estados Unidos. Ante tales condiciones, la ausencia de protección familiar y estatal incrementa su vulnerabilidad y los obliga a desplazarse de forma forzada, a huir (Camargo, 2014, p. 20).

Además, si se ha insistido en que la violencia es factor causal de la migración de niños y niñas, su inclusión en este análisis debe ser referenciada con cautela. En particular, los contextos de violencia barrial, la actuación del crimen organizado y las propias dinámicas familiares donde la violencia verbal y física contra los niños y niñas está presente, son factores desencadenantes, más no los únicos y determinantes.

Un estudio desarrollado por Orozco y Yansura (2014) indica que los niños y niñas son impulsados a salir de sus contextos familiares y barriales por fuerzas paramilitares, pandillas, carteles, traficantes de armas y extorsionistas (Orozco y Yansura, 2014, p. 16). Esta situación expresa ciertamente escenarios complejos y contradictorios, explicados por la suma de factores económicos e institucionales ya evidenciados en este artículo.

La producción social de la violencia a escala regional no debe situarse desde lecturas unicausales, que derivan en abordajes basados en la seguridad y el resguardo de los territorios, enfoque en el que muchas ocasiones se perjudican personas que más bien constituyen víctimas del accionar social e institucional presente.

En el marco de los procesos mediados por violencias localizadas, destaca el impacto en actores que resultan a menudo invisibilizados en los análisis, por ejemplo, lo que ocurre con las personas LGTBI, niños y jóvenes. La violencia de género es el resultado de la discriminación hacia personas de la población LGTBI perpetrada por parejas o miembros de la propia familia, así como en los entornos comunales y colectivos. Para hacer frente a la discriminación, recurren a la migración como respuesta. Es posible que este tipo de movimientos, frecuentes en los marcos regionales recientes, esté subrepresentada en los registros institucionales y no se haya logrado identificar con claridad su volumen por las condiciones de riesgo y anonimato en el que establecen sus rutas migratorias. Su estudio y análisis debe formar parte de agendas de investigación futuras. 
Por su parte, la región ha mostrado impactos derivados de procesos "de desarrollo", tales como la construcción de proyectos de generación eléctrica, explotación minera, producción agroindustrial, que han supuesto el desplazamiento de poblaciones hacia otros sitios fuera de sus lugares de origen. En este movimiento se desestructuran las formaciones familiares y comunales, incidiendo en las dinámicas de salida de muchos de sus miembros, entre los que se cuentan los niños y niñas. El impacto de fenómenos socioambientales y de factores asociados con los efectos de la acción de la naturaleza también expone la estabilidad de las poblaciones y las obliga a movilizarse de manera forzada. En estas dinámicas quienes mayores impactos experimentan sin lugar a duda son los niños y niñas.

No menos importante resulta el efecto que en la región centroamericana ha tenido el conflicto entre autoridades mexicanas y actores del crimen transnacional organizado. Como se señaló anteriormente, la denominada guerra contra las drogas ha tenido un impacto en la violencia y la inseguridad en la región (Shannon, 2014). Las políticas, que incluyen aumentos de recursos para la identificación de la droga en México, la deportación de personas extranjeras, han incrementado la inseguridad y la violencia. El aumento de la violencia crea un clima de temor que impulsa la población fuera de sus localidades de origen.

\section{Sobre las motivaciones}

Las motivaciones, por su parte, recogen trayectorias e historias de vida de los niños y niñas, que determinaron la decisión de migrar. En dicha dimensión intervienen los recursos que conforman la agencia del sujeto y lo llevan a plantearse estrategias para salir, en la mayor parte de los casos sin sus padres o encargados legales, y buscar objetivos y horizontes nuevos fuera de sus hogares y comunidades. Los estudios académicos, la respuesta a las intimidaciones o amenazas, el reencuentro con sus familiares, las agresiones o acoso sexual o alguna razón médica son parte de las motivaciones que encuentran los niños y niñas para aventurarse en el viaje migratorio.

La crisis existió para las autoridades migratorias de Estados Unidos y México, porque reveló su impericia en la atención mesurada de la presencia de cientos de miles de niños en los territorios fronterizos. Antes que eso, se ensayaron respuestas vinculadas con la seguridad, el reforzamiento de esquemas de control y de aseguramiento, donde no importaba ni la persona ni el ejercicio de sus derechos. Para el niño y niña implicó enfrentarse a situaciones nuevas, la ampliación de otras que ya experimentaba en sus contextos de origen (como la exclusión, la invisibilización y el riesgo permanente dada la violencia), para las cuales ensayó posibilidades de reacción de acuerdo con sus condiciones etarias, sociales y culturales, y la conformación de nuevos lazos afectivos a lo largo de las rutas migratorias y de los territorios de destino. 
La decisión de migrar no se produce en el vacío. Se necesitan ciertas condiciones individuales que trascienden una mera lógica de impulso frente a situaciones que se experimentan. El caso de Kevin, el niño hondureño del documental comentado al inicio de este artículo, resulta paradigmático. Con sus recursos individuales, interpuso objetivos por cumplir en su viaje migratorio a Estados Unidos. El imaginario de las oportunidades con el cual identificó a este país y su propia respuesta ante el contexto familiar en Honduras inculcó en el niño una serie de potencialidades para enfrentar los riesgos durante la travesía y el paso por la frontera norte. No fue un agente actuando a la libre formando parte de un cardumen humano incontrolado y expansivo. Su forma de proceder estuvo siempre definida, porque lo llevó a reconocer rutas, establecer relaciones de amistad temporales con otros pares iguales a él, para compartir el viaje y las circunstancias propias de sus decisiones.

La consideración sobre la agencia para analizar las circunstancias de los niños y niñas migrantes, no supone, sin embargo, renunciar a calificar su situación como extrema y atendible por quienes deben hacerlo. No se trata de una especie de adultización del mundo del niño y niña, en el marco de contextos caracterizados justamente por la precariedad institucional, el desajuste familiar y el quiebre comunitario, donde impera la violencia como forma de convivencia cotidiana.

La ruptura de las formas que tenían sentido para el niño y niña (como la escuela y, agregaríamos el entorno familiar) le dejan en posición desventajosa para el cumplimiento de sus derechos humanos más elementales. Ante la situación, reaccionan y ejecutan, pero siguen siendo personas susceptibles de ver la vida como niños y niñas que son. Por eso, lo que ven, lo que experimentan desde que salen de sus contextos familiares, comunitarios, en el tránsito y en el destino, les marcará sus vidas, sus memorias, sus cuerpos, para siempre.

Al calor de la ya citada coyuntura de 2014, muchos discursos otorgaban características de incontenibilidad, el desbordamiento, la magnitud a los flujos migratorios de niños y niñas centroamericanas. Uno de los artículos periodísticos de entonces titulaba "Marea de niños desborda a Estados Unidos", haciendo referencia a supuestas oleadas diarias que comprometían el accionar de autoridades y personas dedicadas al tratamiento humanitario de la situación. Tales lecturas no particularizaban en la persona, sino en su participación como parte de un proceso complejo por su intensidad y tamaño. Tras estos análisis se trazaba un proceso de invisibilización de la agencia del sujeto. En este marco, analizar lo que se ha producido en la región con un grupo significativo de personas cuyas experiencias les han atravesado sus mentes, emociones y cuerpos implica relevar sus biografías, considerar sus propias verbalizaciones sobre lo que sienten, experimentan y viven.

El estudio realizado por Camargo (2014) para la oficina del Alto Comisionado para los Refugiados (ACNUR) en México fue profuso en revelar la voz de los 
niños y niñas migrantes identificados en aquel país detectados en albergues y sitios de aseguramiento (detención) donde eran llevados antes de proceder con su devolución a sus países de origen. En estas voces es posible "observar" las motivaciones, las causas, los miedos, pero sobre todo los desafíos que los mismos niños y niñas se plantean como respuesta y estrategia frente a la acción e impacto de factores de naturaleza estructural. Algunos testimonios relevantes ejemplifican de mejor manera esta relación entre la estructura y la agencia del sujeto:

- "Mejor me fui, hace mucho que mi familia dejó de ser mi protección" (Jeremías, 15 años, Guatemala).

- "Yo quería ir a estudiar a Kansas City donde está un tío mío. Le hablé y le pregunté si me apoyaba para el viaje y me dijo que si. Allá dejé la escuela porque tenía un amigo que andaba en pandilla y pensaron que yo también. Así que me amenazaron y mejor me fui ... pues yo quiero seguir estudiando" (Nancy, 16 años, El Salvador).

- "En El Salvador las cosas se han tranquilizado un poco, pero donde vivo yo todo sigue igual. Los mareros mandan y yo voy con miedo a la escuela y en mi casa. A una amiga de la clase reciente la violaron y yo tengo miedo que me pase a mí. Por eso le pedí a mi papá que me llevara con él. Espero donde está él (en Estados Unidos) las cosas estén más tranquilas" (Yuni, 16 años, El Salvador).

- "Felipe, un hondureño de 15 años, relató que le pusieron dedo en el barrio sin saber por qué. Él dice que porque era amigo de la novia de un marero y un día le habló. Por ello le dieron consigna. Dejó la escuela, dejó a su novia y avisó a su padre en Florida que necesitaba ir. Llama la atención que nunca dicen huir. Su padre aceptó de mala gana pero le puso un guía. En una carretera en Chiapas, México, un oficial de la policía federal le tendió línea porque no pudo darle más que 300 pesos mexicanos que le había dado el coyote para que fuera repartiendo, y más adelante fue aprehendido por migración".

- "El mismo oficial me dijo que porque yo había sido parte de una pandilla era casi imposible que me dieran asilo aquí ... pero yo nunca hice nada con ellos, y para entrar ellos me amenazaron, no fue por gusto, y es por eso que me vine, porque no quiero estar con ellos" (Luis G, 16 años, El Salvador) (Camargo, 2014).

Las anteriores voces representan tan solo una parte de una polifonía mucho más amplia. Son indicativas, eso sí, de decisiones, motivaciones, prácticas de los mismos niños y niñas migrantes y que ponen de manifiesto su actuación frente a procesos familiares, comunitarios y estructurales de donde provienen. Son parte de esas migraciones que no pueden ser denominadas como incontroladas y no pensantes. Tienen objetivos y saben moverse entre los riesgos, advertirlos y tratar de responderlos en consecuencia.

\section{Reflexiones finales}

Las consideraciones expuestas a lo largo de este artículo evidencian la necesidad de considerar a los niños y niñas migrantes como actores sobre los que se deben plantear desafíos en el marco de procesos regionales en 
transcurso y todavía no resueltos. Justamente, la coyuntura de crisis migratoria se produjo en el marco de una serie de dinámicas políticas, geoestratégicas e institucionales de mayores alcances, en las cuales los aspectos de seguridad de Estado y la homogeneización de todos los actores de la migración impusieron impactos que todavía al día de hoy se continúan manifestando. Los flujos de niños y niñas migrantes pusieron en discusión los marcos de referencia en el ejercicio de los derechos humanos por parte de autoridades mexicanas y estadounidenses. Pero sobre todo, colocaron en otra dimensión los efectos agudos de las medidas adoptadas por ambos países en la presunta guerra contra los actores del crimen transnacional organizado, como el narcotráfico, el tráfico de armas y el tráfico de personas.

Ciertamente, la coyuntura puso en evidencia percepciones y valoraciones de orden público, mediático e institucional, que han quedado al descubierto por erradas, cortoplacistas e invisibilizadoras de los rasgos específicos que toma la migración del niño desde que sale del contexto de origen, pasa por los espacios del tránsito y llega a su destino. La historia de Kevin permite observar bajo un lente distinto, las dimensiones de una condición que debería ser analizada tal como es. Los niños y niñas migrantes, tan traídos y llevados en los medios de comunicación y, en ciertos análisis, son personas con capacidad de toma de decisiones, por lo que deben trascenderse los discursos adulto céntricos desde los cuales los miramos cuando tratamos de entenderlos. Al menos dos dimensiones resultan importantes de indicar en este sentido:

Por un lado, la consideración de niños y niñas no acompañados niega las posibilidades de constitución de redes migratorias que estos actores van tejiendo a lo largo del viaje junto con otros pares o con personas adultas. Resulta entonces evidente que debería matizarse dicha denominación y empezar a articular explicaciones más concretas sobre los procesos de construcción de autonomía que están tras las lógicas de esta migración. Ciertamente, esta discusión no solo tiene que ver con los procesos migratorios, porque está articulada a análisis mucho más amplios sobre la desestructuración y el quiebre mostrado por instituciones organizadoras de lo social y lo cotidiano: el mundo de la familia, el mundo del trabajo y el de la escuela son entornos que en Centroamérica están experimentando una serie de transformaciones y deben ser analizados dados sus impactos en los procesos de integración de los niños y las niñas en los ámbitos cultural y estructural.

Por otro lado, resulta evidente que asistimos a un ejercicio mediático de homogeneización y estandarización de los flujos migratorios, ensayado en otros momentos para advertir dinámicas migratorias complejas y sus impactos en los países de tránsito y destino. En este marco, cualquier referencia a los niños y niñas migrantes contenía en su elaboración las mismas categorías utilizadas para abordar la migración adulta a nivel intra y extra regional: las oleadas, el volumen, el desborde, son algunos de los rasgos con los que la opinión pública y los imaginarios vincularon la coyuntura y quedaron con esa idea. 
Desde este punto de vista, fueron negadas las posibilidades de advertir características particulares de los niños y niñas migrantes. En algunos buenos trabajos revisados para la elaboración de este estudio y realizados para organismos internacionales, organizaciones sociales y civiles de defensa de las personas migrantes hubo acercamientos a perfiles y características, pero siempre desde el dato demográfico tradicional. Cuando hubo posibilidad, se afinó la mirada al origen étnico de las poblaciones, identificando las agrupaciones indígenas guatemaltecas como principal rasgo.

Sin embargo, no hubo referencias concisas a las migraciones de niños y niñas afrodescendientes (por ejemplo, si desde las poblaciones garífunas hondureñas se están identificando flujos migratorios con estas características) o como en el caso del estudio de Camargo (2014), que señaló problemas metodológicos para abordar niños y niñas migrantes provenientes de la diversidad sexual. Este último aspecto es importante de señalar en vista de los impactos que han tenido políticas de desprotección para dicha población en la mayor parte de países de la región.

El desafío para la academia y el nivel institucional es claro. En el primer caso, la utilización de la discusión entre agencia y estructura, es necesario para desmontar imaginarios sobre un sujeto amenazante, incontrolado, invasivo, pero que requiere abordajes y tratamientos de acuerdo con sus particularidades como sujeto de derechos. En el caso institucional, la cuestión es más seria y profunda, y requiere cambiar los enfoques privativos que han permeado los abordajes para la contención de la coyuntura, como ha quedado evidente en las presentes reflexiones. Esto para el caso de las autoridades migratorias de los países de destino y tránsito implicados. En el caso de las organizaciones sociales que trabajan en el acompañamiento y la defensa de derechos, es importante trabajar elementos de incidencia en materia de identificación de las particularidades que presentan estos flujos migratorios en la región.

\section{Referencias bibliográficas}

ACNUR, OIT, OIM, UNICEF. (2013). Niños, niñas y adolescentes migrantes. América Central y México. San José. Recuperado de http://costarica.iom. int/public/pdf/ninez_america_latina.pdf

Acuña González, Guillermo E. (2006). Migración y trabajo infantil y adolescente: una aproximación para la construcción de una agenda regional. Ponencia en III Foro de Organizaciones No Gubernamentales de Iberoamérica. Montevideo.

Acuña González, Guillermo E. (2010). Migración de niños, niñas y adolescentes, derechos humanos y trabajo infantil. Los nuevos actores en la migración y su incorporación al mercado de trabajo en la región: algunos elementos para su análisis. San José: Defensa de Niñas y Niños / Internacional. 
Recuperado de http://www.dnicostarica.org/wp-content/themes/sahifa/ plataforma-subregional/Investigaciones-realizadas-por-la-plataforma-subregional/EstudioMigraci\%C3\%B3nNNADHTIPlataforma.pdf

Bakewell, Oliver; Castles, Stephen; Jonsson, Gunvor \& Vezzoli, Simona. (2009). Mobility and human development. Oxford: University of Oxford.

Brettel, Caroline. (2002). The Individual/Agent and Culture/Structure in the History of the Social Sciences. Social Science History, 26(3), 429-442.

Camargo, Abdel. (2014). Arrancados de raíz. Causas que originan el desplazamiento transfronterizo de niños, niñas y adolescentes no acompañados y/o separados de Centroamérica y su necesidad de protección internacional. México: ACNUR.

CAMMINA. (julio, 2014). Comunicado público. Visita de Diálogo e intercambio sobre niñez migrante. Washington. Recuperado de http://observatoriocolef. org/Articulo/343

Camissa, Rebecca. (Directora). (2009). Wich Way Home [Documental]. Estados Unidos: GOOD y White buffaloentertaiment.

Carreño Figueras, José. (8 de junio de 2014). Marea de niños desborda a Estados Unidos. Excelsior. Recuperado de http://www.excelsior.com.mx/nacional/2014/06/08/963945

Castañeda, Alejandra. (15 de julio de 2014). Menores migrantes que viajan sólos. Entre el refugio y el rechazo. CNN México, Opinión. Recuperado de http://mexico.cnn.com/opinion/2014/07/15/opinion-menores-migrantesque-viajan-solos-entre-el-refugio-y-el-rechazo

Centro Nacional de Justicia para inmigrantes. (2014). Niños inmigrantes sin acompañantes reportan serios abusos por parte de funcionarios de Estados Unidos durante la detención. Washington.

Comisión Económica para América Latina y el Caribe (CEPAL). (2007). La juventud en Iberoamérica. Tendencias y urgencias. Buenos Aires. CEPAL.

Ceriani Cernadas, Pablo. (2015). Niñez y migración en Centro y Norte América: causas, políticas, prácticas y desafíos. San Francisco, California: Center forgender and refugeestudies / Universidad Nacional de Lanús.

CNN en español. (2014). La realidad de los niños y niñas migrantes que buscan cruzar la frontera de Estados Unidos [Reportaje]. Recuperado de http:// cnnespanol.cnn.com/2014/06/17/la-realidad-de-los-ninos-centroamericanos-que-buscan-cruzar-la-frontera-de-ee-uu/ 
Cruz, Tania. (Febrero, 2005). Migración y Juventudes. Reflexiones en torno a los riesgos que implican estar en la frontera sur de México. Ponencia en Seminario Las migraciones transfronterizas, San José.

Escobar Sartí, Carolina. (2008). Los pequeños pasos en un camino minado. Migración, niñez y juventud en Centroamérica y el sur de México. Guatemala: Consejería de Proyectos.

Fukunaga, Kary. (Director). (2009). Sin Nombre [Película]. Estados Unidos / México: Focus Feature.

Galen Carpenter, Ted. (2014). The Child Migrant Crisis is Just the Latest Disastrous Consequence of Americas Drug War. Recuperado de http://www. cato.org/publications/commentary/child-migrant-crisis-just-latest-disastrous-consequence-americas-drug-war

García, María del Carmen. (2011). Violencia y ética. A propósito de la inmigración irregular y los derechos humanos en la frontera sur de México. En Daniel Villafuerte Solís \& María del Carmen García Aguilar (coords.), Migración, seguridad, violencia y derechos humanos. Lecturas desde el sur. México: Editorial Porrúa.

Márquez Covarrubias, Humberto. (2014). La voz de los actores. Un soplo de vida digna para los caminantes centroamericanos en México. Entrevista con Alejandro Solalinde. Migración y desarrollo, 12, 173-196.

Orozco, Manuel \& Yansura, Julia. (2014). Understanding Central American Migration: the Crisis of Central American Child Migrants in Context. Washington: Inter-american dialogue.

Quesada Diez, Diego. (Director). (2013). La Jaula de Oro [Película]. México: Animal de Luz Films, Kinemascope Films, Machete Producciones.

Ramírez Parra, Eugenia. (Octubre, 2003). Migración de menores de edad en el Soconusco, Chiapas. Ponencia en Primer Coloquio Internacional Migración y Desarrollo. Transnacionalismo y nuevas perspectivas de integración, Zacatecas.

Rivera Farfán, Carolina. (2011). El trabajo de niñas, niños y adolescentes guatemaltecos en el Soconusco, Chiapas. En Daniel Villafuerte Solís \& María del Carmen García Aguilar (coords.), Migración, seguridad, violencia y derechos humanos. Lecturas desde el sur. México: Universidad de Ciencias y Artes de Chiapas / Miguel Ángel Porrúa.

Sanmartín, Anna. (Octubre, 2010). El vínculo migración y desarrollo, ¿Una oportunidad para impulsar estrategias de participación transnacional desde las asociaciones de migrantes? Ponencia en III Jornadas migratorias, justicia 
y ciudadanía. Madrid: Instituto de Filosofía, Consejo Superior de Investigaciones Científicas.

Saraví, Gonzalo. (2009). Juventud y sentidos de pertenencia en América Latina: causas y riesgos de la fragmentación social. Revista CEPAL, (98), 47-65.

Sassen, Saskia. (15 de junio 2010). De ciudadanos a meros sobrantes. Clarín. Recuperado de http://www.clarin.com/opinion/ciudadanos-meros-sobrantes_0_281372008.html

Shanon, Amy. (1 de julio de 2014). El éxodo de niños de Centroamérica y México. Crisis urgente con raíces profundas. Alianza Nacional de Comunidades Caribeñas y Latino Americanas (NALACC). Recuperado de http://www.nalacc.org/es/immigrant-children-urgent-crisis-with-deep-roots/

Sin Frontreras. (2005 ). México y su frontera sur. México: Sin Fronteras.

Vennet, Fabienne. (2006). Niñez migrante: invisibles y excluidos. Ponencia en Foro de Niñez migrante repatriada no acompañada en la frontera Norte: Hacia una agenda legislativa y una política pública integrales, Tijuana.

Villafuerte, Daniel. (2011). Políticas de seguridad y migración transnacional en la frontera sur de México. En Daniel Villafuerte Solís \& María del Carmen García Aguilar (coords.), Migración, seguridad, violencia y derechos humanos. Lecturas desde el sur. México: Universidad de Ciencias y Artes de Chiapas / Miguel Ángel Porrúa. 